\title{
Nuchal Cord
}

National Cancer Institute

\section{Source}

National Cancer Institute. Nuchal Cord. NCI Thesaurus. Code C113153.

An umbilical cord that has become looped around the fetus's neck from movement in utero. 